\title{
A Further Comprehensive Review on the Phytoconstituents from the Genus Erythrina
}

\author{
Mohammad Musarraf Hussain \\ Department of Pharmacy, Faculty of Life and Earth Sciences, Jagannath University \\ Dhaka-1100, Bangladesh
}

(Received: August 22, 2019; Accepted: January 28, 2020; Published: January 30, 2020)

\begin{abstract}
Erythrina is a significant source of phytoconstituents. The aim of this review is to solicitude of classification, synthesis, and phytochemicals with biological activities of Erythrina. In our previous review on this genus (Hussain et. al., 2016a) fifteen species (Erythrina addisoniae, E. caribeae, E. indica, E. lattisima, E. melanacantha, E. mildbraedii, E. poeppigiama, E. stricta, E. subumbrans, E. veriagata, E. vespertilio, E. velutina, E. zeberi, E. zeyheri and E. americana) have been studied and 155 molecules with chemical structures were reported. A further comprehensive review was done upon continuation on the same genus and thirteen species (E. abyssinica, E. arborescens, E. berteroana, E. burttii, E. caffra, E. coralloids, E. crista-galli, E. fusca, E. herbaceae, E. lysistemon, E. mulungu, E. speciosa and E. tahitensis) of Erythrina have been studied and 127 compounds are reported as phytoconstituents with their chemical structure in this review. Erythrina crista-galli and E. lysistemon consist of highest number of chemical constituents.
\end{abstract}

Key words: Phytoconstituents, classification, Erythrina, leguminoseae, biological properties.

\section{Introduction}

One hundred and ten species of shrubs and trees are present under the genus Erythrina (Family: Leguminosae). A total thirteen medicinal plants have been reviewed comprehensively in this study (Hussain et. al., 2016a). E. abyssinica is a medium sized tree, 5-15 $\mathrm{m}$ in height, well branched and leaves are compounded. E. arborescens (Himalayan coral tree) is a deciduous tree growing up to $5 \mathrm{~m}$ tall; branches and trunk have been covered with prickles. E. berteroana (Coral bean) is a shrub like tree $(10 \mathrm{~m}$ tall) and trunk $(48 \mathrm{~cm})$ having red flower. E. burttii is a flowering plant found in Kenya and Ethiopia. $E$. caffra is a medium to large sized deciduous tree, 9-12 $\times 7-11 \mathrm{~m}$ in height. E. coralloides (Nacked coral tree) is a fast growing deciduous tree $30 \mathrm{ft}$ in height with bright red flowers in early spring. E. crista-galli is a deciduous tree growing to $15-20 \mathrm{ft}$ tall having dark furrowed bark and green leaflets. E. fusca (Coral bean) is deciduous tree having spiny bark and light orange flowers. E. herbaceae (Coral bean) is a shrub (20 ft in height) but sometimes it may be smaller and leaves are semi deciduous 6-8 inch long. $E$. lysistemon is a small to medium sized deciduous and lovely tree $(10 \mathrm{~m}$ in height) with brilliant and spreading crown red flowers. E. mulungu (Ornamental tree) is a medicinal plant native to Brazil and South America. E. speciosa is a tree native to Brazil, cultivated, and introduced populations in Africa and India. E. tahitensis is a species of legume (Family: Fabaceae) endemic to French Polynesia. Species of Erythrina have been used as a traditional medicine in sedative, febrifuge, anti-asthmatic, antiepileptic, convulsion, fever, inflammation, bacterial infection, insomnia, helminthiasis, cough, cuts, and wounds (Kumar et. al., 2010).

Classification of Erythrina alkaloids: Depending on skeleton, two types of Erythrina alkaloids are present i) Erythrinane (1) consisting of a 6,5,6,6 Indoloisoquinoline and ii) Homoerythrinane (2)

Correspondence to: Mohammad Musarraf Hussain. Email: mmhussain@pharm.jnu.ac.bd and m.musarraf.hussain@gmail.com, ORCID: 0000-0001-9270-0474.

DOI: https://doi.org/10.3329/bpj.v23i1.45321 
containing 6, 5, 7,6 Indolobenzazepine skeleton (Figure 1). On the basis of D ring present in the skeleton, Erythrina alkaloids are classified as aromatics $(3,4,5,6,7,8,9,10)$, hetero-aromatics $(\mathbf{1 1}, \mathbf{1 2}, \mathbf{1 3})$ and non-aromatics $(\mathbf{1 4}, \mathbf{1 5})$ (Amer et. al., 1991; Barton et. al., 1970; Barton et. al., 1974;

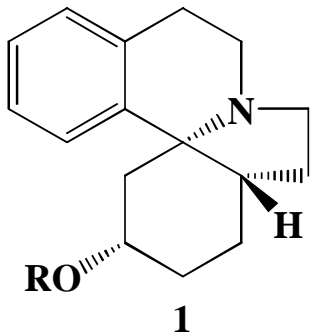

Figure 1. Erythrina alkaloids depending on skeleton

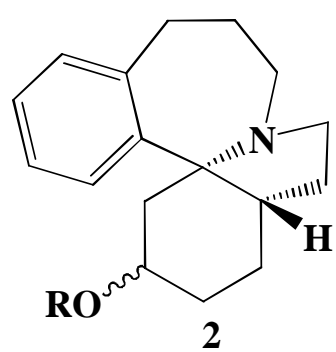
2012) (Figure 2).

$3, \mathbf{R}^{1}=\mathbf{R}^{2}=\mathrm{CH}_{2}, \mathrm{n}=1$ : Erythraline $4, R^{1}=R^{2}=H, n=1$ : Erysopine $5, R^{1}=\mathrm{CH}_{3}, \mathrm{R}^{2}=\mathrm{H}, \mathrm{n}=1$ : Erysovine 6, $\mathrm{R}^{1}=\mathrm{H}, \mathrm{R}^{2}=\mathrm{CH}_{3}, \mathrm{n}=1$ : Erysodine $7, \mathrm{R}^{1}=\mathrm{R}^{2}=\mathrm{CH}_{2}, \mathrm{n}=2$ : Schelhammeridine<smiles>COC(=O)c1cc2c(cn1)CCCN1CCC2=CC[C@H](OC)C1</smiles>

12: Holidine

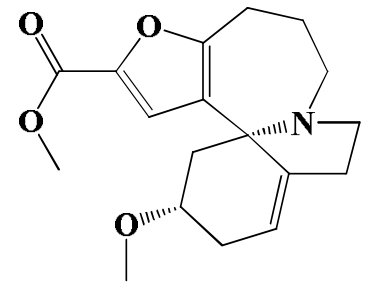

13: Selaginoidine

Carmack et al., 1951; Folkers et. al., 1939, 1940a, 1940b, 1940c, 1937; Garin-Aguilar et. al., 2000; Maier et. al., 1097, 1999; Soto-Hernandez et. al.,

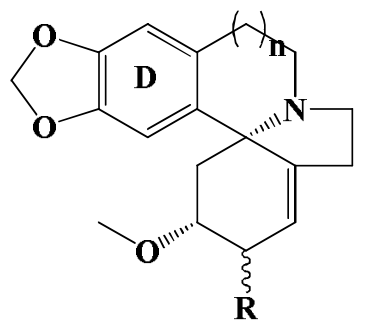

$8, \mathrm{R}=\mathrm{H}, \mathrm{n}=1$ : Erythramine 9, $\mathrm{R}=$ beta- $\mathrm{OH}, \mathrm{n}=1$, Erythratine 10,R $=H, n=2$, Schelhammericine<smiles>COC(=O)c1cc2c(cn1)CCN1CC=C[C@H]2C1</smiles>

11: Erymelanthine<smiles>CO[C@H]1C=CC2=CCN3CC[C@H]4COC(=O)C=C4[C@]23C1</smiles>

14: Erythroidine<smiles>CO[C@H]1CC=C2CCN3CCC4=C(C(=O)OC4)[C@]23C1</smiles>

15: Cocculolidine

Figure 2. Classification of Erythrina alkaloids on the basis of D ring present.

Biosynthesis of Erythrina alkaloids: Angiosperms is the key source of alkaloids, but it can be exists in animals as well as in microorganisms, insects, plants, and marine organism. Alkaloids are secondary metabolites that consist of secondary, tertiary, and quaternary nitrogen atoms in their molecular formula. Alkaloids have been used as medicines and in the recovery of poison. Metabolically, alkaloids are playing a significant function in the physiology at organism and plants. Three types of alkaloids are presents such as true, proto, and pseudo-alkaloids. Different bio-synthetic pathway of alkaloids had been offered, but the objective of this review is the demonstration of a bio- 
synthetic pathway of Erythrina alkaloid. According to the Maier and Soto-Hernandez (Maier et. al., 1997, 1999; Soto-Hernandez et. al., 2012) Erythrina alkaloid, $\Delta^{3}$-Erythratinone (24) can be synthesized from the starting material (S)-Norreticuline (16) through synthesis of few intermediate products such as Norisosalutaridine (17), Noramurine (18), Neospirinic cation (20), and Diallylic cation (23) (Scheme 1).

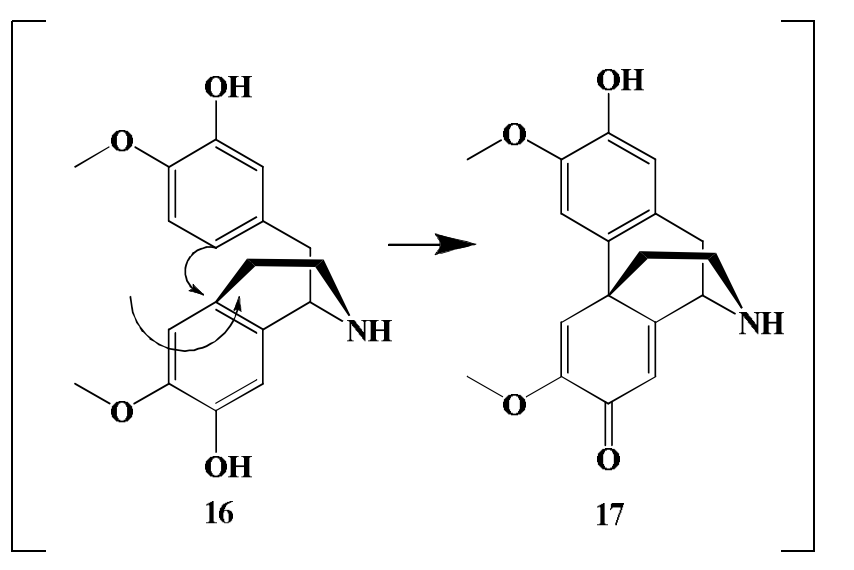

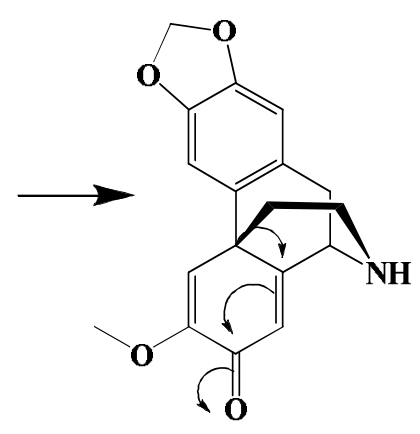

18<smiles>CCNCCc1cc(O)c(OC)cc1-c1cc2c(cc1CCNCC)OCO2</smiles>

21<smiles>CCCCCCC</smiles><smiles>CC[NH+]=CCc1cc2c(cc1-c1cc(OC)c(O)cc1CC)OCO2</smiles>

20
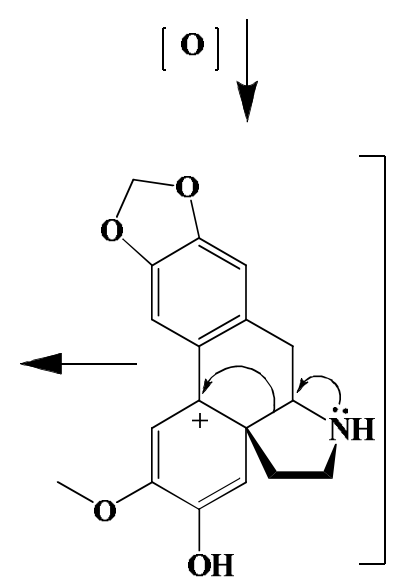

19<smiles>COC1=CC(c2cc3c(cc2CCNCC[SiH2]C)OCO3)C(c2cc3c(cc2CCN2CCNCC2)OCO3)=CC1O</smiles>

22<smiles></smiles>

Scheme 1. Synthetic pathway of Erythrina alkaloids 
Chemical synthesis of the Erythrinane alkaloid: Erythrinane alkaloid Erysodienone (30) can be synthesized form the precursor of $S$ Norprotosinomenine (25) with the synthesis of some intermediate compounds such as Neoproaporphine
(26), (Z)-2,12-Dimethoxy-6,9-dihydro-5H-dibenzo[d,f]-azonine-3,11-diol (27), Dibenzazonine (28), and Dibenzazonine dione (29) (Soto-Hernansez et. al., 2012; Cui et. al., 2009) (Scheme 2).

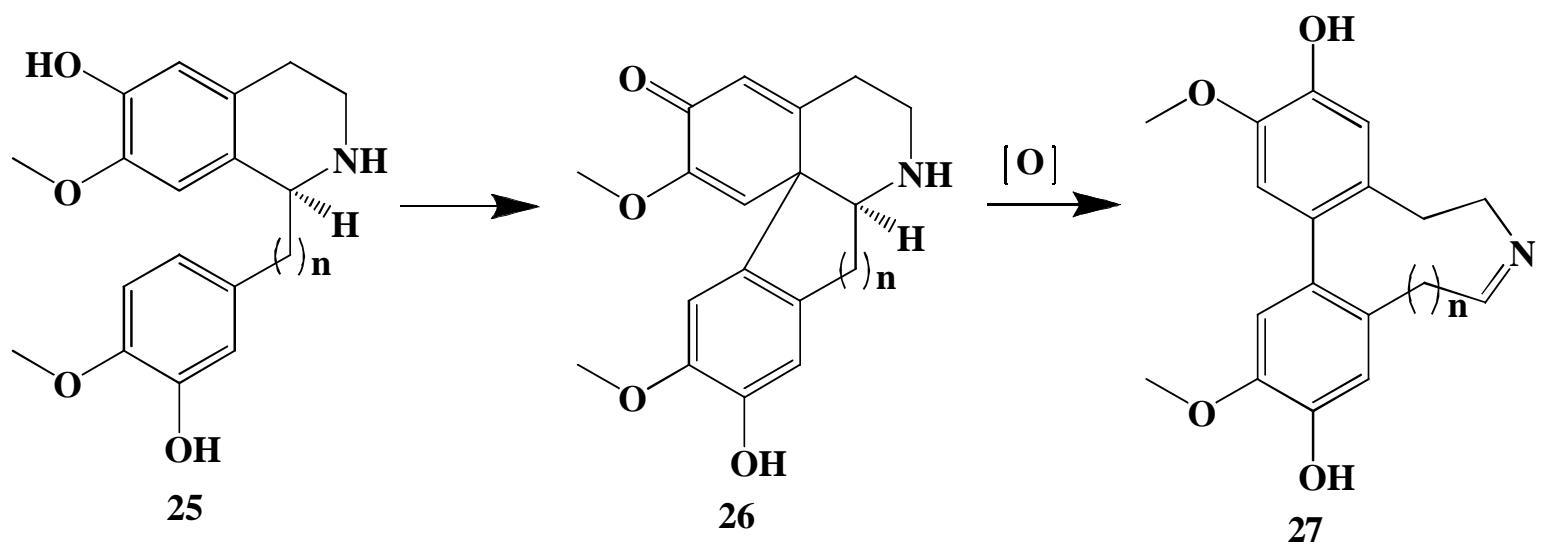<smiles>CCC1=CC(=O)C(OC)=C[C@@]12CCNc1cc(OC)c(O)cc12</smiles>

30

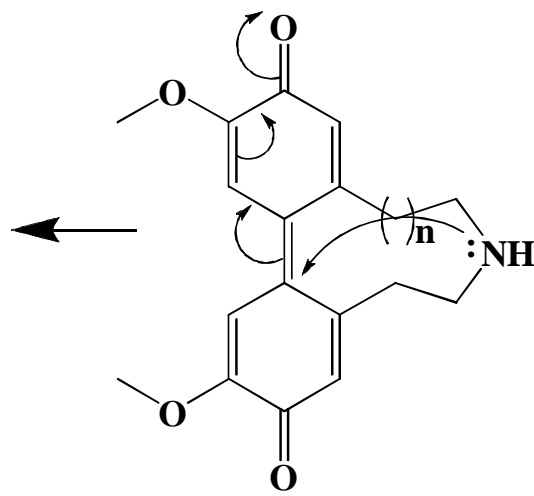

29
$\downarrow^{\mathbf{H}_{2}}$<smiles>CCCCNCCc1cc(O)c(OC)cc1-c1cc(OC)c(O)cc1C</smiles>

Scheme 2. Synthesis of Erythrinane alkaloid.

Phytoconstituents: A total thirteen species of Erythrina have been studied and ninety six molecules (31-127) with chemical structures are reported in this review as bioactive compounds (Figure 3-15).

Erythrina abyssinica: A bunch of chemical compounds such as Abyssinone A (31), Abyssinone B (32), Abyssinone C (33), Abyssinone D (34), Abyssinone V (35), Erythribyssins A (36),
Erythribyssins B (37), Erythribyssins C (38), Eryvarin K (39), Neurautenol (40), Erybraedin B (41), 3,9-Dihydroxy-4-prenyl-[6aR:11aR]pterocarpan (42), Folitenol (43), Erybraedin D (44), Erysubin E (45), Erybraedin C (46), Phaseollidin (47), Sophora pterocarpan A (48), Erythrabyssin-II (49), and Erystagallin (50) were reported from $E$. abyssinica (Figure 3) (Cui et. al., 2008; Kabenei et. al., 2011; Nguyen et. al., 2009). 
Erythrina arborescens: Eight phytochemicals were reported from E. arborescens for example (+)Erysotramidine (51), (+)-11- $\beta$-Hydroxyerysotrine (52), (+)-Erythrascine (53), (+)-Erytharbine (54), (+)-
Erysophorine chloride (55), (+)-Erysodinophorine hydroxide (56), (+)-Erysopinophorine hydroxide (57), and (+)-Isoerysopinophorine hydroxide (58) (Figure 4) (Amer et. al., 1991).<smiles>CC(C)=CCc1cc(C2CC(=[W])C3=C(CC(O)C=C3O)O2)cc(CC=C(C)C)c1O</smiles><smiles>CC1(C)Oc2c(O)cc(/C=C/C(=O)c3ccc(O)cc3O)cc2C(O)C1O</smiles>

$\mathbf{R}_{2}$

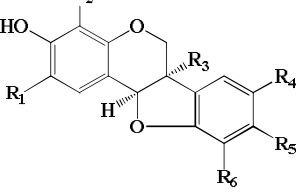

36: $\mathrm{R}_{1}=\mathrm{R}_{2}=\mathrm{R}_{4}=\mathrm{II}, \mathrm{R}_{3}=\mathrm{OCH}_{3}, \mathrm{R}_{5}=\mathrm{CH}_{3}, \mathrm{R}_{6}=$ Prenyl 37: $\mathbf{R}_{1}=\mathbf{R}_{2}=\mathbf{R}_{3}=\mathbf{R}_{4}=\mathbf{R}_{5}=\mathbf{H}, \mathbf{R}_{6}=\mathbf{C H O}$

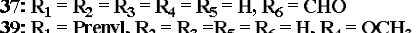

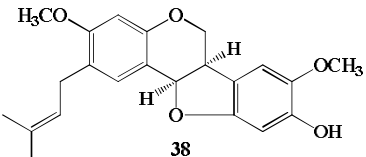<smiles>CC1(C)C=Cc2cc3c(cc2O1)OC[C@H]1c2ccc(O)cc2O[C@H]31</smiles>

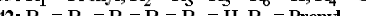

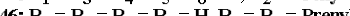

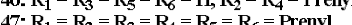
. $R_{1}=R_{2}=R_{3}=R_{1}=R_{1}=R_{6}=R_{4}=17 y$ 48: $R_{1}=R_{2}=R_{3}=R_{5}=R_{6}=H, R_{4}=$ Prenyl 49: $R_{1}=R_{6}=$ Prenyl, $R_{2}=R_{3}=R_{4}=R_{5}=H$
50: $R_{1}=R_{6}=$ Prenyl, $R_{2}=R_{4}=H, R_{3}=O H, R_{5}=C_{3}$
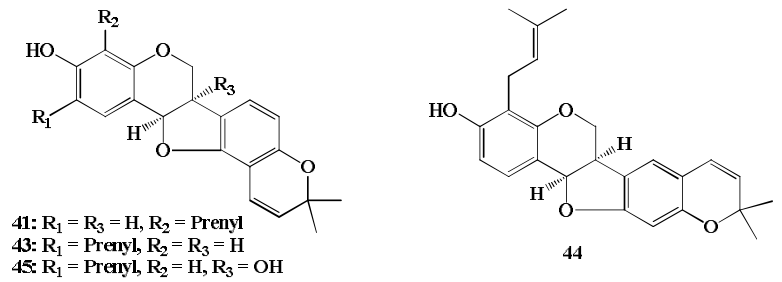

Figure 3. Molecules from Erythrina abyssinica<smiles>CC=CCC12C(C=CC)=CC(=O)N1CCc1cc(OC)c(OC)cc12</smiles><smiles>CCC1=CCN2C[C@H](O)c3cc(OC)c(OC)cc3C12CCCOC</smiles><smiles></smiles><smiles>COc1cc2c(cc1OC)[C@]13NNC=CC1=CC(=O)N3C=C2</smiles>

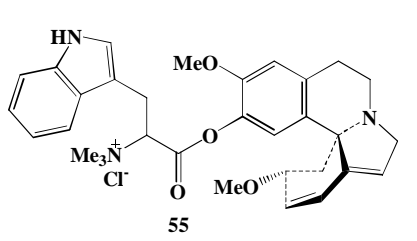

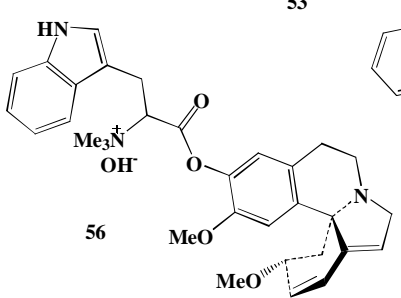<smiles>CO[C@H]1C=C[C@H]2CCN3CCc4cc(OC(=O)C(Cc5c[nH]c6ccccc56)N(C)O)c(O)cc4[C@@]23C1</smiles><smiles>C=CC1=CCN2CCc3cc(O)c(OC(=O)[C@@H](Cc4c[nH]c5ccccc45)[14C](=O)OC)cc3[C@@]12CC</smiles>

Figure 4. Chemical constituents from Erythrina arborescens. 
Erythrina berteroana: The reported chemical compounds from this plants are (+)-11Hydroxyerythratidine (59), (+)-Hydroxyerysosalvine (60), (+)-11-Hydroxyerysotine (61), (+)- $\alpha$ -
Erythroidine (62), (+)- $\beta$-Erythroidine (63), (+)-8Oxo- $\alpha$-erythroidine $\quad(64), \quad$ and $(+)-8-O x o-\beta-$ erythroidine (65) (Figure 5) (Amer et. al., 1991).
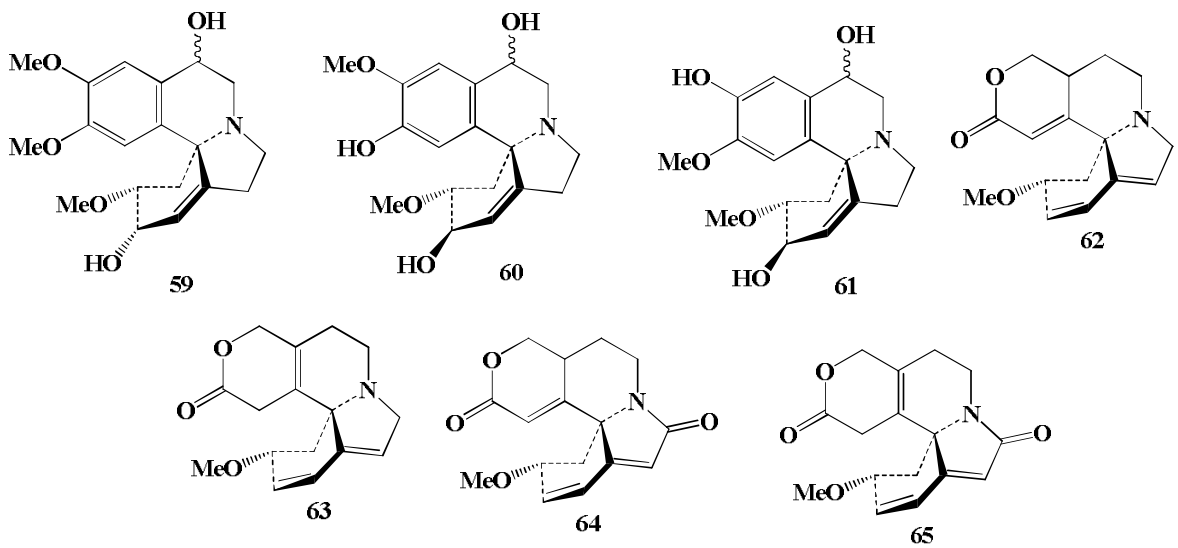

Figure 5. Phytoconstituents from Erythrina berteroana
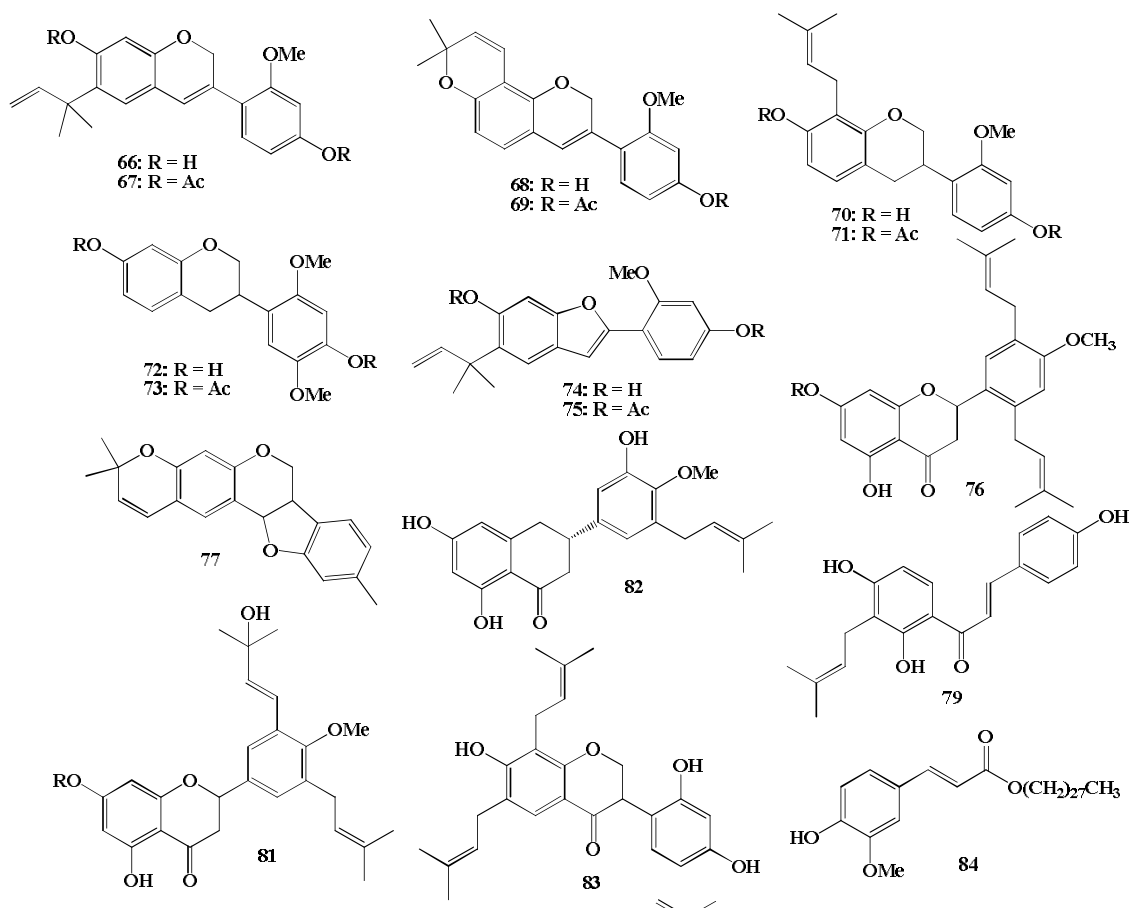

8
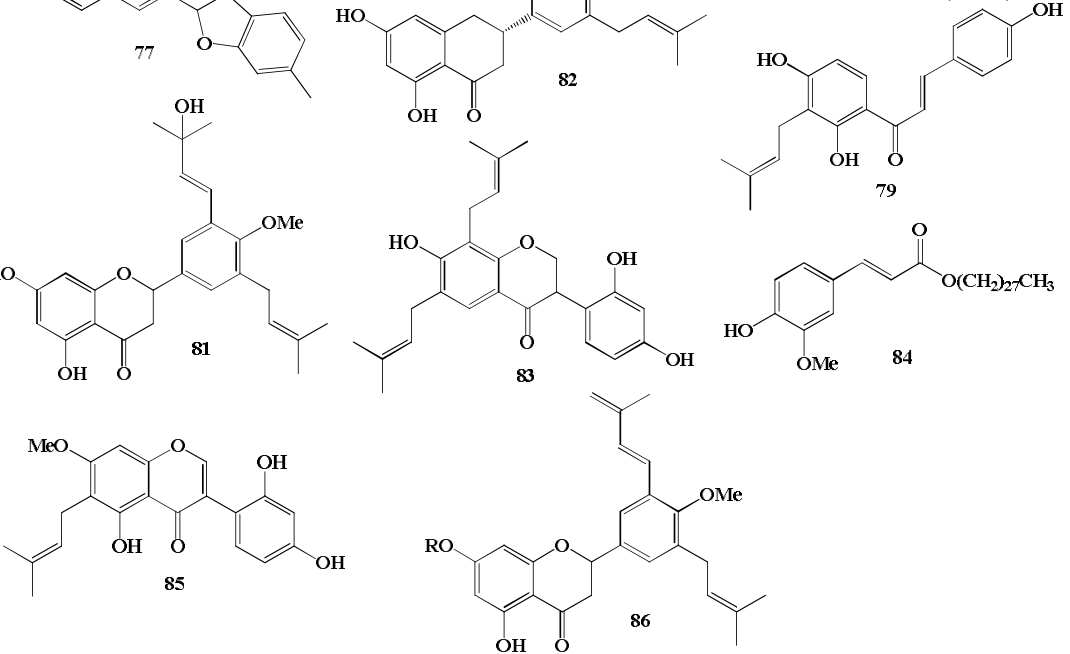

Figure 6. Secondary metabolites from Erythrina burttii 
Erythrina burttii: The isolated bioactive moieties from this medicinal plants are Abyssinone V (35), Bruttinol-A (66), Burtinol-A diacetate (67), Bruttinol-B (68), Bruttinol-B acetate (69) Bruttinol-C (70), Bruttinol-C diacetate (71), Eryvarin-H (72), Eryvarin-H diacetate (73), Bruttinol-D (74), Bruttinol-D diacetate (75), Abyssinone-V-4'-methyl ether (76), Calopocarpin (77), Alpinumisoflavone (78), Erythrabissin-II (49), Isobavachalcone (79), Phaseollidin (47), Phaseollin (80), Burttinone (81), Neurautenol (40), 4'-O-Methylsigmoidin (82),
Bidwillon A (83), Erythrinasinate (84), 7'-OMethyllutenone (85), Burttinonedehydrate (86), 8Prenyllutenone (87), 3-O-methylcalopocarpin (88), and Genistein (89) (Figure 6) (Yenesew et. al., 2002, 1998, 2003, 2012; Hussain, 2018).

Erythrina caffra: Four secondary metabolites such as (+)-Erysotrine (90), (+)-Erysodine (91), (+)8-Oxo-erysodine (92), and (+)-11-Methoxyerysopine (93) were reported from this plants (Figure 7) (Amer et. al., 1991; Taria et. al., 1994).

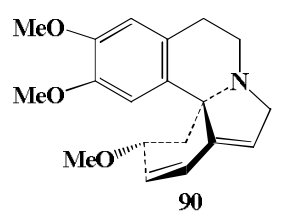

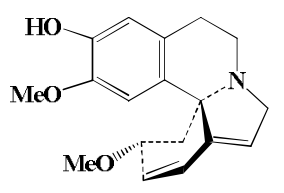

91

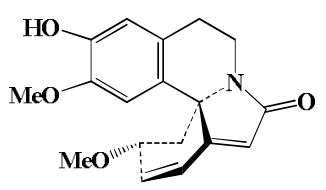

92

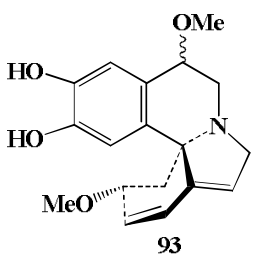

93

Figure 7. Molecules from Erythrina caffra.
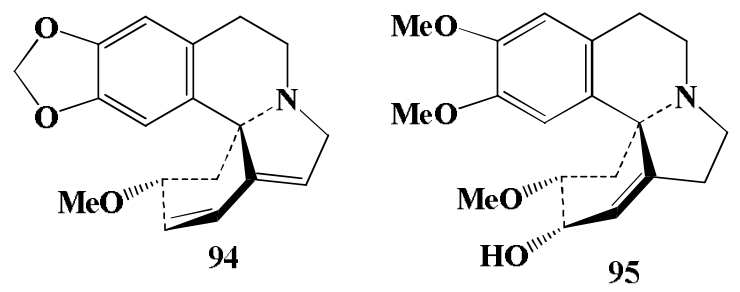

Figure 8. Compounds from Erythrina coralloides.

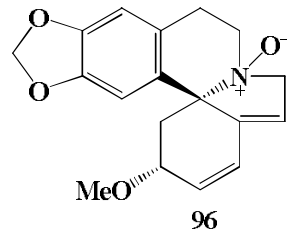<smiles>[R4]C1CN2CC(Br)c3cc4c(cc3C23CC(C)C=CC43)OCO1</smiles>

100: $\mathrm{R}_{1}=\mathrm{II}, \mathrm{R}_{2}=\mathrm{O}$ 101: $\mathrm{R}_{1}=\mathrm{OII}, \mathrm{R}_{2}=\mathrm{II}$ 102: $\mathrm{R}_{1}=\mathrm{OHI}, \mathrm{R}_{2}=\mathrm{O}$

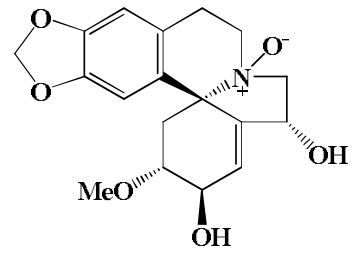<smiles></smiles>

103<smiles></smiles>

98

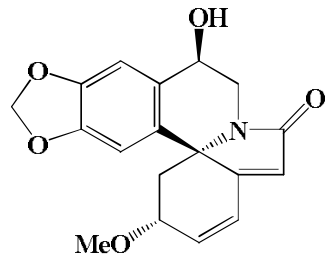

99<smiles>COc1cc2c(cc1OC)[C@]1(CC2)C[C@@H](O)C(O)=CC1CCN</smiles><smiles></smiles>

Figure 9. Constituents from Erythrina crista-galli 


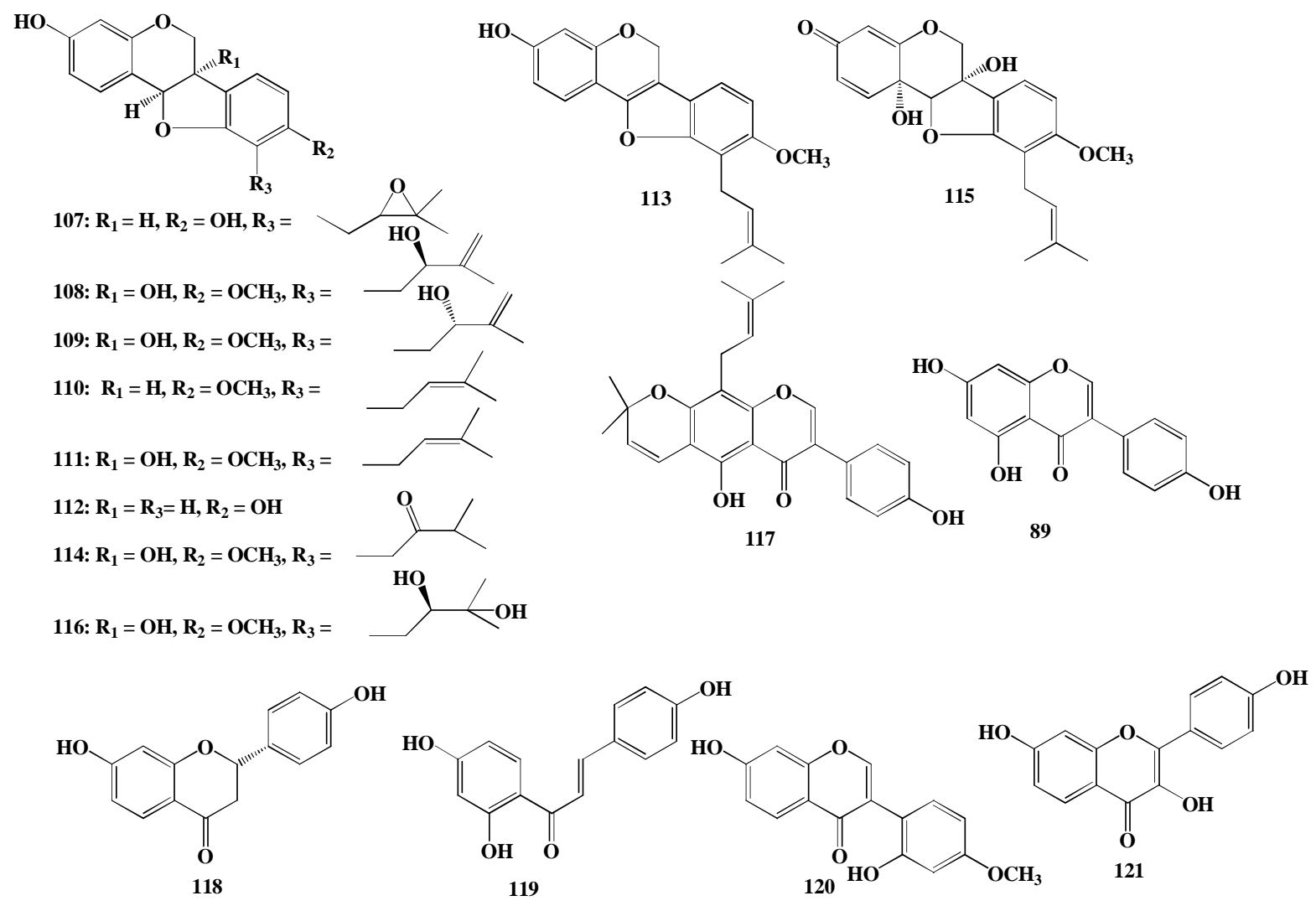

Figure 10. Chemical compounds from Erythrina fusca

Erythrina coralloides: The reported three isolated phytochemicals from this plant are (+)Erythraline (94), (+)-Erysopine (4), and (+)Erythratidine (95) (Figure 8) (Amer et. al., 1991; Soto-Hernandez et. al., 2012; Hussain, 2018; Taria et. al., 1994).

Erythrina crista-galli: A group of chemical moieties having biological properties reported from this plants are Cristanines A (96), Cristanines B (97), Erythratine (98), Crystamidine (99), Erysovine (5), Erythraline (3), 8-Oxo-erythraline (100), Erythrinine (101), 8-Oxo-erythrinine (102), Erythratidine (103), Epi-erythratidine (104), Hypaphorine (105), and Lupeol (106) (Figure 9) (Amer et. al., 1991; Taria et. al., 1994; Miquel-Chavez et. al., 2006).

Erythrina fusca: A set of isolated phytoconstituents from this plant are Erythraline (3), Epi-erythratidine (104), Fuscacarpans A (107), Fuscacarpans B (108), Fuscacarpans C (109), Sandwicensin (110), Erythribyssins A (36),
Erythrabissin I (111), Demethylmedicarpin (112), Eryvarin D (113), Erypoegin (114), Hydroxycristacarpone (115), Orientanol A (116), Scandenone (117), Genistein (89), Liquiritigenin (118), Isoliquiritigenin (119), Vestinone (120), and 3,7,4'-Trihydroxyflavone (121) (Figure 10) (Amer et. al., 1991; Ozawa et. al., 2010; Innok et. al., 2010).

Erythrina herbaceae: The isolated chemical compounds from this plant are Erybacin A (122), Erybacin B (123), 6-formyl-2,2-dimethyl-2Hchromene (124), Eryvariestyrene (125), Glyasperin F (126), Eryvarin H (72), Bidwillol A (127), Phaseollinisoflavan (128), Erythbidin A (129), Phaseollidinisoflavan (130), Eryvarin L (131), and Glabrocoumarone A (132) (Figure 11) (Tanaka et. al., 2010, 2003, 2001).

Erythrina lysistemon: (+)-Erythristemine (133), (+)-11- $\beta$-Methoxyglucoerysovine (134), (+)-11Hydroxyerysovine (135), (+)-11-Methoxyerysovine (136), (+)-Glucoerysodine (137), (+)-11- $\beta$ - 
Methoxyglucoerysodine (138), (+)-Rhamnoerysodine (139), (+)-11- $\alpha$-Hydroxyerysodine (140), (+)-11- $\beta$ Hydroxyerysodine (141), (+)-11- $\beta$-Methoxyerysodine
(142), and (+)-8-Oxo-11- $\beta$-methoxyerythraline (143) are reported from this plant (Figure 12) (Amer et. al., 1991).<smiles>[2H]C([2H])(c1ccc(O)cc1OC)[C@H](Cc1ccc2c(c1)C=CC(C)(C)O2)OC</smiles><smiles>CC(C)=CCc1cc(/C=C/c2ccccc2)c(O)cc1O</smiles>

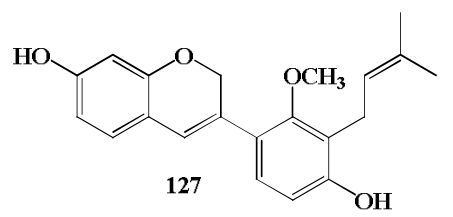

$\mathrm{HO}$<smiles>CC(C)=CCc1c(O)ccc(C2COc3ccccc3C2)c1O</smiles><smiles>C=Cc1c(OC(C)(C)C)ccc(C(=O)Cc2ccc(O)cc2O)c1O</smiles>

123<smiles>CC1(C)C=Cc2c(O)ccc(C3COc4cc(O)cc(O)c4C3=O)c2O1</smiles><smiles>COc1cc(C2=Cc3ccc(O)cc3OC2)c(OC)cc1O</smiles><smiles>CC1(C)C=Cc2c(ccc(C3COc4cc(O)ccc4C3)c2O)O1</smiles><smiles>CC1(C)C=Cc2c(O)ccc(C3COc4cc(O)ccc4C3)c2O1</smiles><smiles>COc1cc(-c2cc3ccc(O)cc3o2)c(OC)cc1O</smiles><smiles>CC1(C)C=Cc2c(O)ccc(-c3cc4ccc(O)cc4o3)c2O1</smiles>

Figure 11. Phytochemicals from Erythrina herbaceae

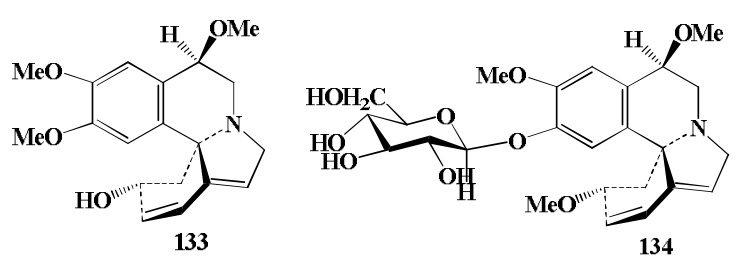

133

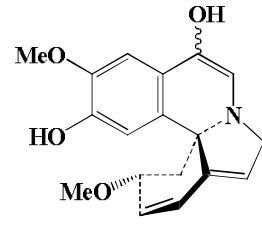

135<smiles>CCC1CCN2C=C(OC)c3cc(O)c(O)cc3C12</smiles>

136

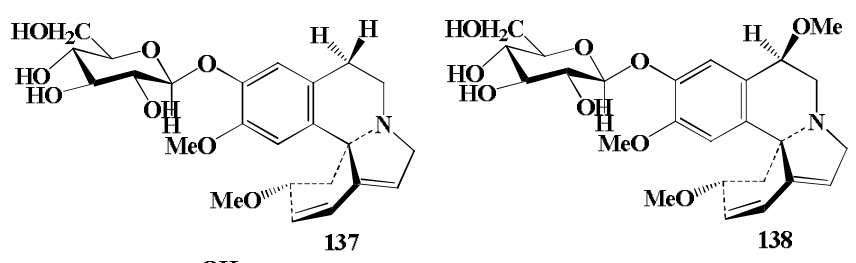<smiles>CC(C)C1CCN2CC(O)c3cc(O)c(O)cc3C12</smiles><smiles>C#CC1CCN2CC(O)C3C=C(O)C(O)C(CC)C3C12</smiles><smiles>COc1cc2c(cc1O)C(C)CN1CC=C(C)C1C2CO</smiles>

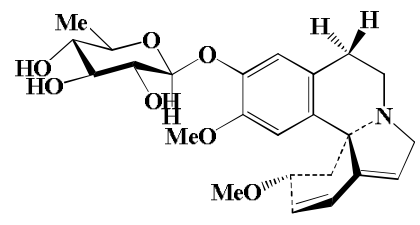

Figure 12. Phytoconstituents from Erythrina lysistemon

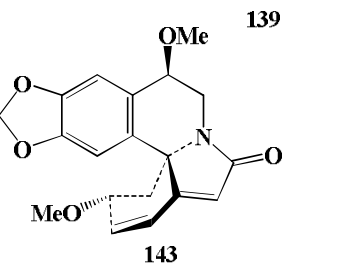


Erythrina mulungu: Seven reported molecules from this plant are Erysotrine (90), Erysovine (5), Hypaphorine (105), (+)-Erythravine (144), (+)-11- $\alpha-$ hydroxy-erythravine (145), (+)-Erysotrine $\mathrm{N}$-oxide
(146), and (+)-Erythrartine $N$-oxide (147) (Figure 13) (Amer et. al., 1991; Yenesew et. al., 1998; Sarragiotto et. al., 1981).

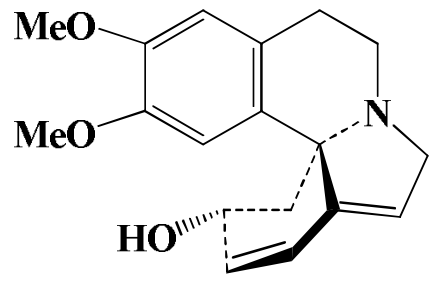

144

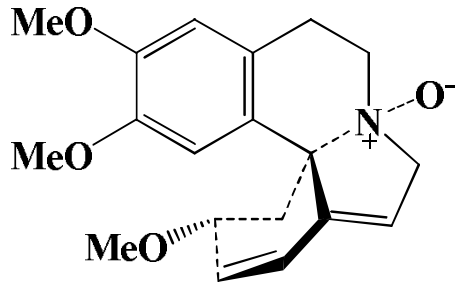

146

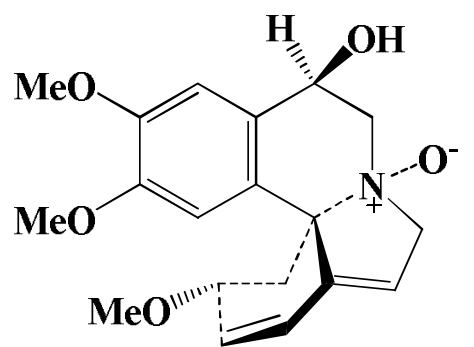

147

Figure 13. Chemical compounds from Erythrina mulungu.<smiles>C=C1C=C[C@@H](C)C[C@]12c1cc(OC)c(OC)cc1[C@@H](O)CN2C</smiles>

148<smiles>[R10]Oc1cc2c(cc1[R10])[C@]13CCN2CC=C1C=CC([R16])C3</smiles>

149: $\mathrm{R}_{1}=\mathrm{H}, \mathrm{R}_{2}=\mathrm{R}_{3}=\mathrm{Me}$ 150: $R_{1}=R_{3}=M e, R_{2}=H$ 151: $R_{1}=R_{3}=H, R_{2}=M e$ 152: $R_{1}=R_{2}=C_{2}, R_{3}=H$

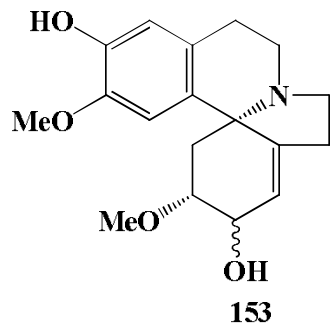

153<smiles>COc1cc2c(cc1O)C(Cc1ccc(O)c(O)c1)NCC2</smiles>

154

Figure 14. Chemical compounds from Erythrina speciosa.

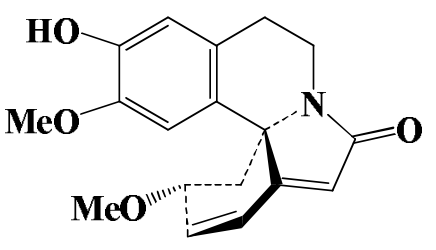

155<smiles>COc1cc2c(cc1O)C13CCN1CC(=O)c1ccc(cc1OC)CC23</smiles>

156<smiles>CO[C@H]1C=C[C@@H]2CCN3CC(=O)c4cc(O)c(O)cc4[C@@]213</smiles>

157

Figure 15. Chemical compounds from Erythrina tahitensis.

Erythrina speciosa: The elucidated chemical compounds from this plant are Erysotrine (90), Erythrartine (148), Erysodine (149), Erysovine (150), Erythsonine (151), E-erythrocarina (152), Eerythsotin (153), and Nororientaline (154) (Figure 14) (Faria et. al., 2007).
Erythrina tahitensis: (+)-8-Oxoerysodine (155), (+)-11-Oxo-Erysovine (156), and (+)-11-Oxoerysopine (157) were isolated as bioactive compounds from this plant (Amer et. al., 1991). 
Biological properties: Medicinal plants are the pioneer source of biologically active constituents (Hussain 2019a 2019b, 2018; Hussain et. al., 2010, 2011, 2008, 2016b; Billah et. al., 2013; Ismail et. al., 2010). As an indigenous medicine, many species of Erythrina have been used as eyewashes, wounds, pain relief of arthritis, and relaxants agents (GarinAguilar et. al., 2000). The pharmacological properties of the derived molecules from the medicinal plants studied in this review are summarized in Table 1.

Table 1. Biological properties of the elucidated derivatives from the medicinal plants.

\begin{tabular}{|c|c|c|}
\hline Phytoconstituents & Biological properties & References \\
\hline (+)-Erythraline and Erythrinine & Radio-labeled activity & Maier et. al., 1999 \\
\hline Abyssinone V & Antimicrobial (Plasmodium falciparum) & Kabenei et. al., 2011 \\
\hline $\begin{array}{l}\text { Abyssinone A, Abyssinone B, Abyssinone } \\
\text { C, and Abyssinone D }\end{array}$ & Cytotoxic and PTP1B inhibition & $\begin{array}{l}\text { Cui et. al., 2008; Nguyen } \\
\text { et. al., } 2009\end{array}$ \\
\hline Bruttinol-A, Bruttinol-B, and Bruttinol-D & Anti-plasmodial and radical scavenging & Yenesew et. al., 2012 \\
\hline Erythraline, Erythrinine, and Hypaphorine & $\begin{array}{l}\text { LPS induced NO production and cell } \\
\text { viability in RAW } 264.7 \text { macrophase }\end{array}$ & Ozawa et. al., 2010 \\
\hline $\begin{array}{l}\text { Fuscacarpans-A, Fuscacarpans-B, and } \\
\text { Fuscacarpans-C }\end{array}$ & Antibacterial, antiplasmodial, and cytotoxic & Innok et. al., 2010 \\
\hline Erybacin A and Erybacin B & $\begin{array}{l}\text { Antibacterial (Staphylococcus aureus) and } \\
\text { bactericidal (MRSA) }\end{array}$ & Tanaka et. al., 2010 \\
\hline $\begin{array}{l}\text { Abyssinone-V-4'-methyl ether and } \\
\text { Alpinumisoflavone }\end{array}$ & Estrogenic & Nde et. al., 2012 \\
\hline Genistein & Antimicrobial & Redko et. al., 2007 \\
\hline $\begin{array}{l}\text { (+)-Erythravine, }(+)-11-\alpha \text {-hydroxy- } \\
\text { erythravine }\end{array}$ & Anticonvulsant and anxiolytic & Rosa et. al., 2012 \\
\hline$\beta$-erythroidine & Radio-labeled activity regarding ${ }^{14} \mathrm{C}$-tyrosine & Mantle et. al., 1984 \\
\hline Erysotramidine and Erysotrine $N$-oxide & Anti-oxidant & Tanaka et. al., 2008 \\
\hline Erysodine & DPPH radical scavenging & Juma et. al., 2004 \\
\hline Lupeol & Antiplasmodial & Chukwujekwa et. al., 2016 \\
\hline
\end{tabular}

\section{Conclusion}

A total thirteen species of Erythrina have been studied. Structurally distinctive including numerous diversified molecules have been reported from this genus. Our study explored that Erythrina genus can be an outstanding source of phytochemicals as well as medicinal agents.

\section{References}

Hussain, M.M., Tuhin, M.T.H., Akter, F. and Rshid, M.A. 2016a. Constituents of Erythrina-a potential source of secondary metabolites: A review. Bangladesh Pharm. J. 19, 237-253.

Kumar, A., Lingadurai, S., Jain, A. and Barman, N.R. 2010. Erythrina variegata Linn: A review on morphology, phytochemistry, and pharmacological aspects. Pharmacogn. Rev. 4, 147-152.
Amer, M.E., Shamma, M., and Freyer, A.J. 1991. The tetracyclic Erythrina alkaloids. J. Nat. Prod. 54, 329363.

Barton, D.H.R., Boar, R.B. and Widdowson, D.A. 1970. Phenol oxidation and biosynthesis. Part XXI. The biosynthesis of the Erythrina alkaloids. J. Chem. Soc. Perkin Trans, 9, 1213-1218.

Barton, D.H.R., Potter, C.J. and Widdowson, D.A. 1974. Phenol oxidation and biosynthesis Part XXIII. On the benzyltetrahydroisoquinoline origins of the Erythrina Alkaloids. J. Chem. Soc. Perkin Trans 1346.

Carmack, M., Mackusick, B.C. and Prelog, V. 1951. Erythrina-alkaloide, 2. Mitt Über das Apo- erysodin und das Apo-erythralin. Helv. Chim. Acta, 34, 16011611.

Folkers, K. and Koniuszy, F. 1939. Erythrina alkaloids, III. Isolation and characterization of a new alkaloid, Erythramine. J. Am. Chem. Soc. 61, 1232-1235. 
Folkers, K. and Koniuszy, F. 1940a. Erythrina alkaloids. VII. Isolation and characterization of the new alkaloids, Erythraline and Erythratine. J. Am. Chem. Soc. 62, 436-441.

Folkers, K. and Koniuszy, F. 1940b. Erythrina alkaloids VIII. Studies on the constitution of Erythramine and Erythraline. J. Am. Chem. Soc. 62, 1673-1677.

Folkers, K. and Koniuszy, F. 1940c. Erythrina alkaloids. IX. Isolation and characterization Erysodine, Erysopine, Erysocine and Erysovine. J. Am. Chem. Soc. 62, 1677-1683.

Folkers, K. and Major, R.T. 1937. Isolation of Erythroidine, an alkaloid of curare action from Erythrina americana Mill. J. Am. Chem. Soc. 59, 15801581.

Garin-Aguilar, M.E., Ramirez-Luna, J.E., Soto-Hernandez, M., Valencia, D.T.G. and Martinez-Vazquez, M. 2000. Effect of crude extracts of Erythrina americana Mill. On aggressive behavior in rats. $J$. Ethnopharmacol. 69, 189-196.

Maier, U.H. and Zenk, M.H. 1997. (S)-Norrreticuline is the precursor for the biosynthesis of Erythrina alkaloids. Chem. Commun. 2313-2314.

Maier, U.H., Rodl, W., Deus-Newmann, B. and Zenk., M.H. 1999. Biosynthesis of Erythrina alkaloids in Erythrina crista-galli. Phytochemistry, 52, 373-382.

Soto-Hernandez, M.R. Garcia-Mateos, R., Migual-Chavez, R.S. and Ramos-Valdivia. C. 2012. Erythrina, a potential source of chemicals from the neotropics. Bioactive Compounds Phytomed. 163-184.

Cui, L., Thuong, Fomum, P.T. and Oh, W.K. 2009. A new erythrinan alkaloid from the seed of Erythrina addisoniae. Arch. Pharm. Res. 32, 325-328.

Cui, L., Thuong, P.T., Lee, H.S., Njamen, D., Mbafor, J.T., Fomum, Z.T., Lee, J., Y. Kim, Y.H. and Oh, W.K. 2008. Four new chalcones from Erythrina abyssinica. Planta Medica, 74, 422-426.

Kabenei, J.S., Ndalut, P.K. and Sabah, A.O. 2011. Synergism of artemisinin with abyssinone-V from Erythrina abyssinica (Lam. ex) against Plasmodium falciparum parasites: A potential anti-malarial combination therapy. J. Med. Plant. Res. 5, 13551360.

Nguyen, P.H., Le, T.V.T., Thoung, P.T., Dao, D.T., Ndinteh, D.T., Mbafor, J.T., Kang, K.W. and Oh, W.K. 2009. Cytotoxic and PTP1B inhibitory activities from Erythrina abyssinica. Bioorg. Med. Chem. 19, 6745-6749.
Yenesew, A., Midiwo, J.O., Guchu, S.M., Heydenreich, M. and Peter, M.G. 2002. Three isoflav-3-enes and a 2arylbenzofuran from the root bark of Erythrina burttii. Phytochemistry, 59, 337-341.

Yenesew, A., Midiwo, J.O., Miessner, M., Heydenreich, M. and Peter, M.G. 1998. Two prenylated flavanones from stem bark of Erythrina burttii. Phytochemistry, 48, 1439-1443.

Yenesew, A., Irungu, B., Derese, S., Midiwo, J.O., Heydenreich, M. and Peter, M.G. 2003. Two prenylated flavonoids from the stem bark of Erythrina burttii. Phytochemistry, 63, 445-448.

Yenesew, A., Akala, H.M., Twinomuhwezi, H., Chepkirui, C., Irungu, B.N., Eyase, F.L., Mugisha, M.K., Kiremire, B.T., Johnson, J.D. and Waters, N.C. 2012. The antiplasmodial and radical scavenging activities of flavonoids of Erythrina burttii. Acta Tropica, 123, 123-127.

Taira, Z., Takei, M., Endo, K., Hahimoto, T., Sakiya, Y. and Asakawa, Y. 1994. Marchantin A trimethyl Ether: Its molecular structure and Tubocurarine-like skeletal muscle relaxation activity. Chem. Pharm. Bull. 42, 5256.

Miguel-Chavez, R.S., Soto-Hernandez, M., Terrazas, T. and Kite, G. 2006. Morphology and alkaloidal profile of the seedlings of Erythrina americana Mill and E. coralloides A.DC. Feddes Repertorium, 117, 232-239.

Ozawa, M., Kawamata, S., Etoh, T., Hayashi, M., Komiyama, K., Kishida, A., Kuroda, C. and Ohsaki, A. 2010. Structures of new Erythrinan alkaloids and nitric oxide production inhibitors from Erythrina crista-galli. Chem. Pharm. Bull. 58, 1119-1122.

Innok, P., Rukachaisirikul, T., Phongpaichit, S. and Suksamrarn, A. 2010. Fuscacarpans A-C, new pterocarpans from the stems of Erythrina fusca. Fitoterapia, 81, 518-523.

Tanaka, H., Sudo, M., Kawamura, T., Sato, M., Yamaguchi, R., Fukai, T., Sakai, E. and Tanaka, N. 2010. Antibacterial Constituents from the Roots of Erythrina herbacea against Methicillin-resistant Staphylococcus aureus. Plannta Med.76, 916-919.

Tanaka, H., Etoh, H., Shimizu, H., Uchi, T.O., Terada, Y. and Tateishi, Y. 2001. Erythrinan alkaloids and isoflavonoids from Erythrina poeppigiana. Planta Med. 67, 871-873.

Tanaka, H., Uchi, T.O., Etoh, H., Sako, M., Sudo, M., Sato, M., Fukai, T. and Tateishi, Y. 2003. Erythrinan alkaloids and isoflavonoids from Erythrina poeppigiana. Planta Med. 67, 871-873. 
Sarragiotto, M.H., Filho, H.L. and Marsaioli, A.J. 1981. Erysotrine-N-oxide and erythrartine-N-oxide, two novel alkaloids from Erythrina mulungu. Canadian J. Chem. 59, 2771-2775.

Faria, T.D.J., Cafeu, M.C., Akiyoshi, G., Ferreira, D.T., Galao, O.F., Andrei, C.C., Filho, P., Paiva, M.R.C., Barbosa, A.D.M. and Filho, R.B. 2007. Alkaloids from flowers and leaves of Erythrina speciosa Andrews. Quimica Nova, 30, 525-527.

Hussain, M.M. 2019a. A comprehensive review on the phytoconstituents from six species of the genus Amaranthus. Bangladesh Pharm. J. 22, 117-124.

Hussain, M.M. 2019b. A mini review on the chemical compounds of the genus Acacia. Bangladesh Pharm. J. 22, 235-242.

Hussain, M.M. 2018. A short review on phytoconstituents from the genera Albizzia and Erythrina. Bangladesh Pharm. J. 21, 160-172.

Hussain, M.M., Dastagir, M.G., Billah, A.H.M.M. and Ismail, M. 2011. Alpinum isoflavone from Erythrina stricta Roxb. Bol. Latinoam. Y del Caribe de Plantas Medicinales Y Aromat. 10, 88-90.

Hussain, M.M., Rahman, M.S., Jabbar, A. and Rashid, M.A. 2008. Phytochemical and Biological Investigation of Albizzia lebbek Benth. Bol. Latinoam. $Y$ del Caribe de Plantas Medicinales Y Aromat. 7, 273-278.

Hussain, M.M., Tahia, F. and Rashid, M.A. $2016 \mathrm{~b}$. Secondary metabolites from some species of Albizzia: A review. Bangladesh Pharm. J. 19, 1-8.

Billah, A.H.M.M., Hussain, M.M., Dastagir, M.G., Ismail, M. and Quader, A. 2013. $\alpha$-Spinasterol from Amaranthus spinosus stem. Bol. Latinoam. Bol. Latinoam. Y del Caribe de Plantas Medicinales $Y$ Aromat. 12, 15-17.

Ismail, M., Hussain, M.M., Dastagir, M.G., Billah, M. and Quader, A. 2010. Phytochemical and antimicrobial investigation of Luffa cylindrica. Bol. Latinoam. Y del Caribe de Plantas Medicinales Y Aromat. 9, 327-332.
Hussain, M.M., Mughal, M.M.R., Alam, M.M., Dastagir, M.G., Billah, A.H.M.M. and Ismail, M. 2010. Antimicrobial activity of n-hexane and Ethyl acetate extracts of Erythrina stricta Roxb. Bangladesh J. Microbiol. 27, 65-66.

Nde, C.B.M., Njamen, D., Fomum, S.T., Wandji, J., Simpson, E., Clyne, C. and Vollmer, G. 2012. In vitro estrogenic activity of two major compounds from the stem bark of Erythrina lysistemon (Fabaceae). European J. Pharmacol. 674, 87-94.

Redko, F., Clavin, M.L., Weber, D., Ranea, F., Anke, T. and Martino, V. 2007. Antimicrobial isoflavonoids from Erythrina crista-gallii infected with phomosis SP. Z. Naturfrosch C, 62, 164-168.

Rosa, D.S., Faggion, S.A., Gavin, A.S., Souza, M.A.D., Fachim, H.A., Santos, W.F.D., Pereira, A.M.S., Cunha, A.O.S. and Beleboni, R.O. 2012. Erysothrine, an alkaloid extracted from flowers of Erythrina mulungu Mart. ex Benth: Evaluating its anticonvulsant and anxiolytic potential. Epilepsy Behavior, 23, 205212.

Mantle, P.G. and Coleman, M.J. 1984. Biosynthesis of radiolabelled alkaloids from ${ }^{14} \mathrm{C}$-tyrosine in Erythrina crista-gallii. Phytochemistry, 23, 1617-1618.

Tanaka, H., Hattori, H., Tanaka, T., Sakai, E., Tanaka, N., Kulkarni, A. and Etoh, H. 2008. A new Erythrina alkaloid from Erythrina herbacea. J. Nat. Med. 62, 228-231.

Juma, B.F. and Majinda, R.R.T. 2004. Erythrinaline alkaloids from the flowers and pods of Erythrina lysistemon and their DPPH radical scavenging properties. Phytochemistry, 65, 1397-1404.

Chukwujekwu, J.C., Kock, C.A.D., Smith, P.J., Heerden, F.R.V. and Staden, J.V. 2016. Antiplasmodial, acetylcholinesterase and alpha-glucosidase inhibitory and cytotoxicity properties of Buddleja saligna. South African J. Bot. 106, 101-103. 